\title{
Culturable diversity of Arctic phytoplankton during pack ice melting
}

\section{Supplementary material}

Catherine Gérikas Ribeiro ${ }^{1,2 *}$, Adriana Lopes dos $\operatorname{Santos}^{3,2}$, Priscillia Gourvil ${ }^{4}$, Florence Le Gall ${ }^{1}$, Dominique Marie ${ }^{1}$, Margot Tragin ${ }^{1}$, Ian Probert ${ }^{4}$, Daniel Vaulot $^{1,3}$

${ }^{1}$ Sorbonne Université, CNRS, UMR7144, Team ECOMAP, Station Biologique de Roscoff, Roscoff, France

${ }^{2}$ GEMA Center for Genomics, Ecology \& Environment, Universidad Mayor, Santiago, Chile ${ }^{3}$ Nanyang Technological University, Asian School of the Environment, Singapore.

${ }^{4}$ Sorbonne Université, CNRS, FR2424, Roscoff Culture Collection, Station Biologique de Roscoff, Roscoff, France

*catherine.gerikas@gmail.com

Elementa: Science of the Anthropocene

https://doi.org/10.1525/elementa.401.s1

Date: February 6, 2020 


\section{Supplementary data}

Supplementary data are available at:

https://github.com/vaulot/Paper-2019-Ribeiro-GE-cultures

Supplementary Data S1: File GE_cultures_Tables.xlsx. Sheet Data S1. Strains collected during the Green Edge campaign, including both Amundsen cruise (AM) an ice camp (IC) samples: RCC accession number, taxonomy, respective phylotype grouping, sampling substrate, depth and date, geographic coordinates, and isolation method.

Supplementary Data S2: File GE_cultures_Tables.xlsx. Sheet Data S2. Best BLAST hit for representative 18S rRNA sequences from each phylotype against all GenBank sequences, $\mathrm{PR}^{2}$ sequences and sequences from cultured strains. 


\section{Supplementary Figures}

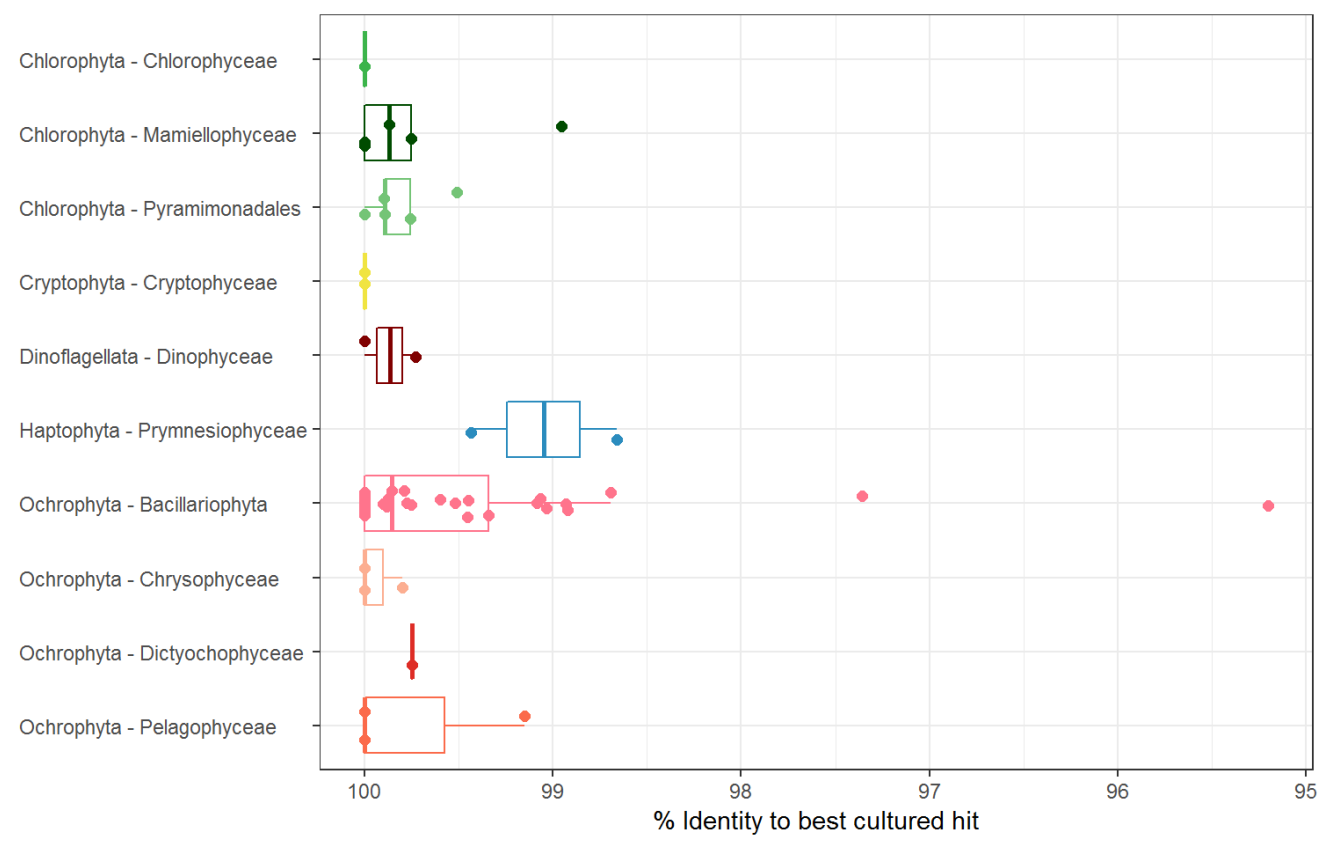

Figure S1. Novelty of phylotypes.

Percentage of similarity of phylotype representative $18 \mathrm{~S}$ rRNA sequence to best BLAST hit from GenBank (see Supplementary Data S2). 


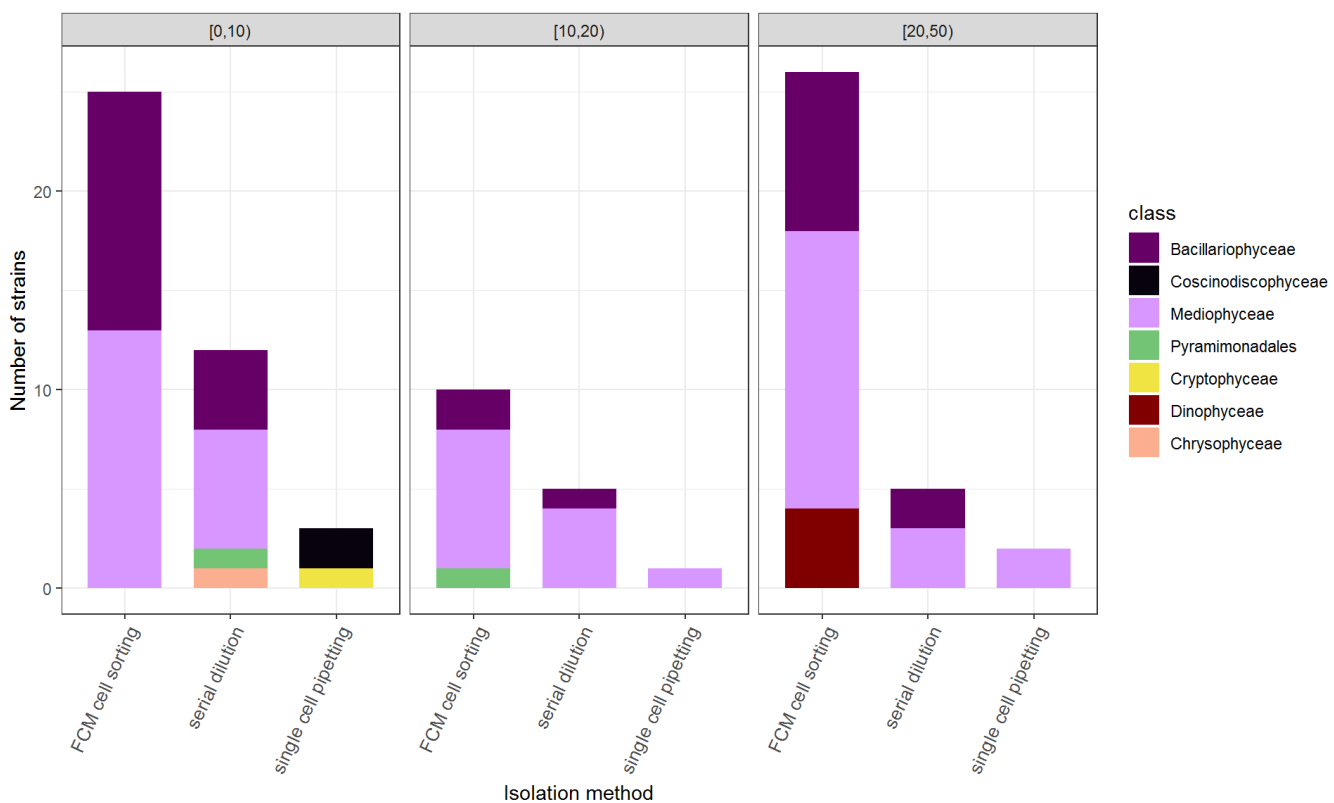

Figure S2. Strains from Amundsen cruise as a function of isolation method and depth.

Strain class distribution for the Amundsen cruise separated according to the method of isolation (cell sorting, serial dilution and single cell isolation) and sampling depth range $(\mathrm{m}, \mathrm{m})$. 


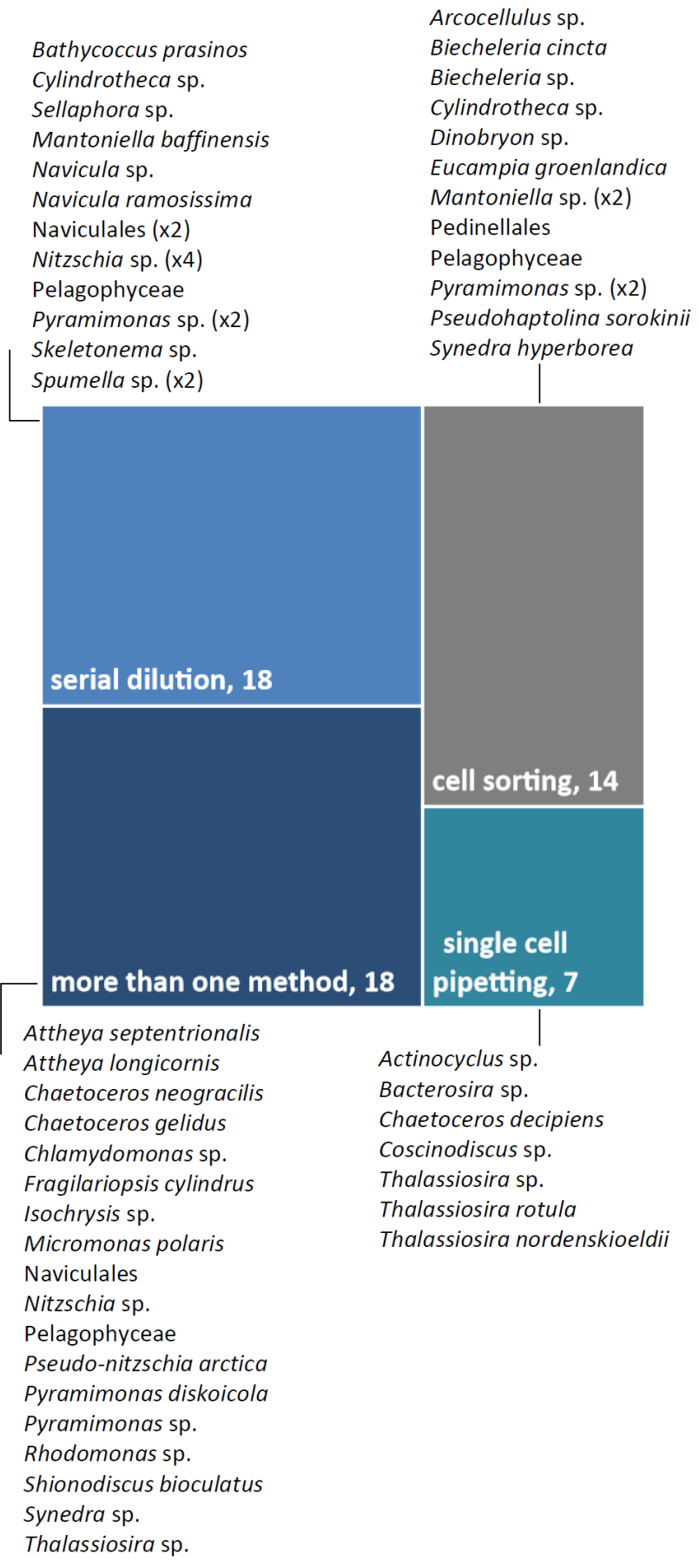

Figure S3. Phylotype as a function of isolation method.

Treemap of the number of strains isolated as function of the isolation method. 

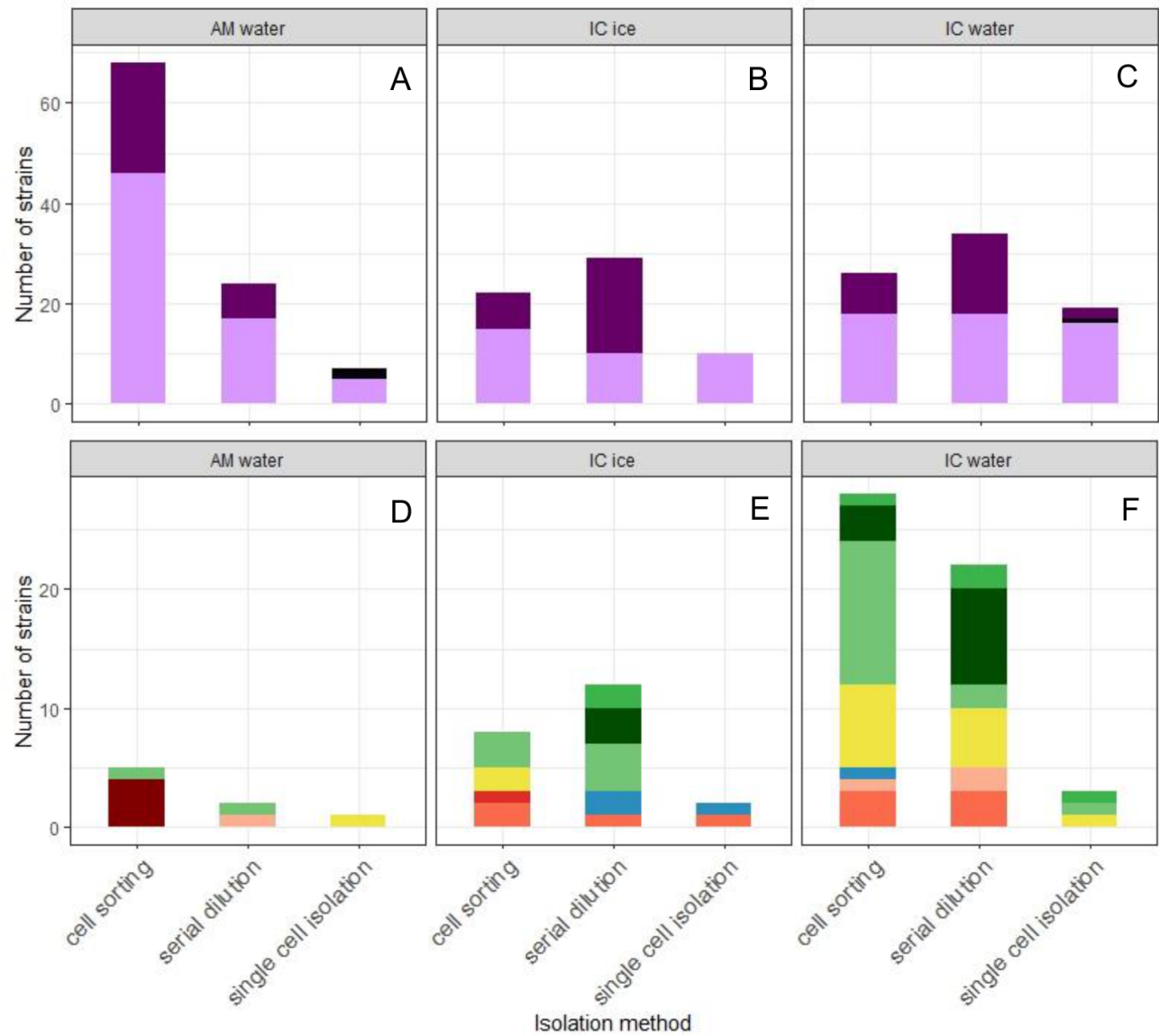

\begin{tabular}{|l|l|l|l|l}
\hline Mediophyceae & Chrysophyceae & Pyramimonadales & Cryptophyceae \\
\hline Bacillariophyceae & Pelagophyceae & Chlorophyceae & Prymnesiophyceae \\
\hline Coscinodiscophyceae & Dictyochophyceae & Mamiellophyceae & Dinophyceae \\
\cline { 2 - 4 }
\end{tabular}

\section{Figure S4. Strains as a function of isolation method and substrate.}

Strain class distribution separated according to the method of isolation (cell sorting, serial dilution, and single cell isolation) and sampling substrate: water samples from the Amundsen cruise, and water and sea ice samples from the Ice Camp for diatoms (top panels) and non-diatoms (bottom panels). 